\section{EVALUATION OF SARS-COV-2 IGG ANTIBODY REACTIVITY IN A MULTI-RACIAL/ETHNIC COHORT OF PATIENTS WITH SYSTEMIC LUPUS ERYTHEMATOSUS}

${ }^{1}$ Amit Saxena*, ${ }^{1}$ Allison Guttmann*, ${ }^{1}$ Mala Masson, ${ }^{2}$ Mimi Y Kim, ${ }^{1}$ Rebecca H Haberman, ${ }^{1}$ Rochelle Castillo, ${ }^{1}$ Jose U Scher, ${ }^{1}$ Kristina K Deonaraine, ${ }^{1}$ Alexis J Engel, ${ }^{1} \mathrm{H}$ Michael Belmont, ${ }^{1}$ Ashira D Blazer, ${ }^{1}$ Jill P Buyon, ${ }^{1}$ Ruth Fernandez-Ruiz*, ${ }^{1}$ Peter M Izmirly*. ${ }^{1}$ Division of Rheumatology, Department of Medicine, New York University School of Medicine, New York, NY; ${ }^{2}$ Division of Biostatistics, Department of Epidemiology and Population Health, Albert Einstein College of Medicine, New York, NY; ${ }^{3}$ NYU Langone Health, New York, NY

\subsection{6/lupus-2021-lupus21century.73}

Background Patients with Systemic Lupus Erythematosus (SLE) represent a unique population at risk for COVID-19 due to underlying immune abnormalities and regular use of immunosuppressant medications. This study was initiated to evaluate for the presence of SARS-CoV-2 IgG antibodies in SLE patients with and without prior COVID-19-related symptoms or COVID-19 RT PCR testing.

Methods A total of 329 patients with SLE from two cohorts, one serially monitored for COVID-19 in Spring 2020 (the Web-based Assesment of Autoimmune, Immune-Mediated and Rheumatic Patients (WARCOV) and one undergoing routine surveillance (NYU Lupus Cohort) were tested for SARS-CoV-2 IgG via commercially available immunoassays processed through hospital or outpatient laboratories between April 29, 2020 and February 9, 2021.

Results Overall, $16 \%$ of 329 patients had a reactive SARSCoV-2 IgG antibody test. Seropositive patients were more likely to be Hispanic. Other demographic variables, lupus-specific factors and immunosuppressant use were not associated with reactivity. Of the 29 patients with prior RT-PCR confirmed COVID-19, 83\% developed an antibody response despite $62 \%$ being on immunosuppressants. Six percent of patients who had symptoms suspicious for COVID-19 but negative concurrent RT-PCR testing developed an antibody response. Twenty-three percent of patients who had COVID19-related symptoms but no RT-PCR testing and 5\% of patients who had no symptoms of COVID-19 developed an antibody response. Among patients initially SARS-CoV-2 IgG positive, the majority maintained reactivity serially. In COVID19 -confirmed patients high percentages had antibody positivity beyond 30 weeks from disease onset, $88 \%$ up to 10 weeks, $83 \%$ up to 20 weeks, and $80 \%$ up to 30 weeks.

Conclusions Most patients with SLE and confirmed COVID19 were able to produce a serologic response despite use of a variety of immunosuppressants. These findings provide reassurances regarding the efficacy of humoral immunity and possible reinfection protection in patients with SLE.

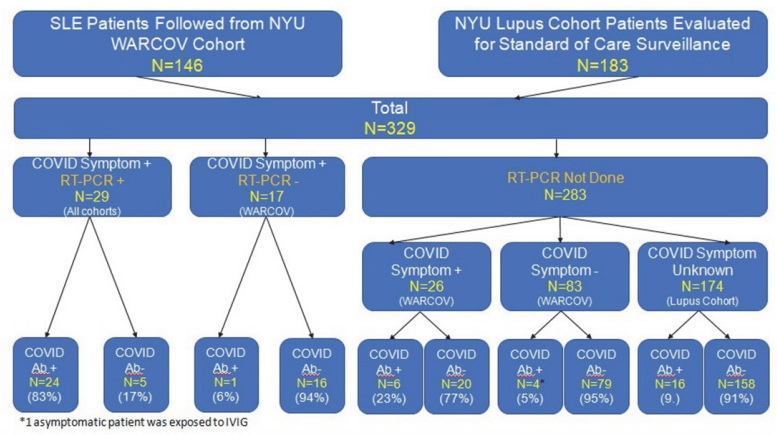

Abstract 1206 Figure 1 SARS-CoV-2 IgG in SLE
Acknowledgments Data presented on behalf of the NYU WARCOV investigators. We thank Leora Horwitz for her assistance with the ICD-10 query at NYU. We also acknowledge Tania Moin and Ranit Shriky for assistance in navigating regulatory matters.

\section{0 - SLE diagnosis}

\section{EFFECT OF ATTRIBUTION ON EXTERNAL VALIDATION OF THE EULAR/ACR SLE CLASSIFICATION CRITERIA}

${ }^{1}$ Nicolai Leuchten, ${ }^{2}$ Karen Costenbader, ${ }^{3}$ Thomas Dörner, ${ }^{4}$ Sindhu R Johnson, 'Martin Aringer*. 'University Medical Center and Faculty of Medicine TU Dresden, Dresden, Germany; '2Brigham and Women's Hospital, Harvard Medical School, Boston, MA, USA; ${ }^{3}$ Charité - Universitätsmedizin Berlin, Berlin, Germany; ${ }^{4}$ University of Toronto, Toronto, ON, Canada

10.1136/lupus-2021-lupus21century.74

Background With their new structure of ever positive antinuclear antibodies (ANA) as an obligatory entry criterion and weighted specific criteria with a cut-off of $\geq 10$, the European League Against Rheumatism/American College of Rheumatology (EULAR/ACR) 2019 classification criteria for systemic lupus erythematosus (SLE) has a sensitivity of $96.1 \%$ and a specificity of $93.4 \%$ in the validation cohort. ${ }^{1,2}$ An analysis of the performance of the individual criteria items

\begin{tabular}{|c|c|c|c|c|c|}
\hline \multirow[t]{2}{*}{ Publication } & \multirow[t]{2}{*}{ Population } & \multicolumn{2}{|c|}{ EULAR/ACR criteria } & \multirow{2}{*}{$\begin{array}{l}\text { Arthritis } \\
\text { Controls }\end{array}$} & \multirow{2}{*}{$\begin{array}{l}\text { ANA } \\
\text { positive }\end{array}$} \\
\hline & & Sensitivity & Specificity & & \\
\hline $\begin{array}{l}\text { Adamichou et al } \\
2019\end{array}$ & Early SLE & $88.6 \%$ & $97.3 \%$ & $37.2 \%$ & $93.6 \%$ \\
\hline Aljaberi et al 2020 & Pediatric SLE & $84.8 \%$ & $82.8 \%$ * & $35 \%$ & $96 \%$ \\
\hline $\begin{array}{l}\text { Aringer et al } 2019 \\
(1,2) \text { (validation) }\end{array}$ & SLE & $96.1 \%$ & $93.4 \%$ & - & $99.3 \%$ \\
\hline $\begin{array}{l}\text { Aringer et al } \\
\text { (derivation) }\end{array}$ & SLE & $98 . \%$ & $96 \%$ & - & $99.6 \%$ \\
\hline Batu et al 2020 & Pediatric SLE & $91.6 \%$ & $88.5 \%$ * & $41.4 \%$ & $98.9 \%$ \\
\hline $\begin{array}{l}\text { Dahlström \& } \\
\text { Sjöwall } 2019\end{array}$ & SLE & $93 \%$ & $73 \%$ * & $38.2 \%$ & $98.2 \%$ \\
\hline $\begin{array}{l}\text { Gegenava et al } \\
2019\end{array}$ & NPSLE & $87 \%$ & $74 \%$ & $16.7 \%$ & $96.3 \%$ \\
\hline Lee et al 2020 & SLE & $97.6 \%$ & $91.4 \%$ * & $31.2 \%$ & $98.8 \%$ \\
\hline Levinsky et al 2021 & Pediatric SLE & $96 \%$ & $89 \% *$ & $35.4 \%$ & $100 \%$ \\
\hline Ma et al 2020 & Pediatric SLE & $97.4 \%$ & $98.4 \%$ & $58 \%$ & $100 \%$ \\
\hline Petri et al 2020 & SLE & $90.8 \%$ & $88.6 \%$ * & $33.4 \%$ & $97.7 \%$ \\
\hline $\begin{array}{l}\text { Rodrigues Fonseca } \\
\text { et al } 2019 \text { (BL) }\end{array}$ & Pediatric SLE & $89.3 \%$ & $67.4 \%$ * & $66.3 \%$ & NR \\
\hline $\begin{array}{l}\text { Rodrigues Fonseca } \\
\text { et al (one year) }\end{array}$ & Pediatric SLE & $95.1 \%$ & $58.4 \%$ * & $73 \%$ & NR \\
\hline Rubio et al 2020 & SLE & $94.9 \%$ & NA & NA & $96.3 \%$ \\
\hline Smith et al 2021 & Pediatric SLE & $94 \%$ & $77 \%$ * & $23.3 \%$ & $93.1 \%$ \\
\hline Suda et al & SLE & $92 \%$ & NA & NA & $92 \%$ \\
\hline Teng et al 2020 & SLE & $96.5 \%$ & $90.3 \%$ * & $39 \%$ & $99.5 \%$ \\
\hline Wang et al 2021 & $\mathrm{LN}$ & $95.2 \%$ & NA & $37.2 \%$ & $95.2 \%$ \\
\hline $\begin{array}{l}\text { Whittall Garcia et al } \\
2021\end{array}$ & SLE & $95.7 \%$ & NA & $35 \%$ & $97.1 \%$ \\
\hline
\end{tabular}

Arthritis controls: \% non-SLE control population with joint diseases, NR not reported, NA not applicable, * included into specificity analysis. LN lupus nephritis. 